Neurosurg Focus 11 (6):Article 3, 2001, Click here to return to Table of Contents

\title{
Medical management and adjuvant therapies in spinal metastatic disease
}

\author{
James M. Schuster, M.D., Ph.D., AND M. Sean Grady, M.D. \\ Department of Neurosurgery, University of Pennsylvania, Philadelphia, Pennsylvania
}

\begin{abstract}
Metastatic spinal tumors are an increasingly common and difficult problem encountered by neurosurgeons and orthopedic surgeons. To improve therapies and increase life expectancy for patients with tumors such as those of the breast and prostate, a global, systematic approach is required to maximize the preservation of neurological function, maintenance of spinal stability, and relief of pain, all with the ultimate goal of improved functional capacity and quality of life (QOL). Although radiotherapy and surgery are still the primary therapeutic options, several new adjuvant therapies initially implemented to control pain more effectively have also been shown to reduce overall skeleton-related complications (pathological fractures and hypercalcemia) and may ultimately improve and extend QOL. This more global approach to spinal metastases also includes optimizing each patient's overall medical condition and potential for healing (that is, nutrition), as well as avoiding potential complications associated with metastatic disease (such as deep vein thrombosis), including excessive blood loss in the case of renal metastasis. A thorough knowledge and understanding of these therapeutic adjuvants is required to optimize care and to respond to our increasingly medically knowledgable patient population whose access to prevalent medical information has been increased because of the internet.
\end{abstract}

KEY WORDS • spine • metastasis • medical management • pain

\section{MEDICAL MANAGEMENT AND ADJUVANT THERAPIES IN SPINAL METASTATIC DISEASE}

Because the spine represents the most common site of skeletal metastatic involvement, neurosurgeons and orthopedists commonly encounter cases of spinal metastases. ${ }^{8}$ Spinal metastases often cause severe pain and neurological compromise, which affect functional abilities and QOL. Rational therapeutic decision making requires a thoughtful consideration of the following critical elements: the patient's general medical condition, the tumor type and its radiosensitivity, tumor stage including the patient's life expectancy, previous therapy, neurological condition, time course of symptom onset, extent of spinal involvement including issues of instability, and patient/ family wishes.

Conservatively, at least $30 \%$ of patients with systemic malignancies will develop spinal metastasis over the course of their disease. ${ }^{14,23,28}$ Of the solid primary tumors, breast, lung and prostate are the most prevalent, followed by renal, gastrointestinal, and thyroid tumors. The estimated incidence of spinal involvement based on findings in autopsy studies may be as high as $60 \%$ in breast cancer. ${ }^{11}$ Spinal cord compression is the first sign of prostate cancer in an estimated 12 to $19 \%$ of patients. ${ }^{10,21}$ Increased life expectancy for breast and prostate cancer patients who harbor spinal metastases will influence decisions concerning issues of long-term vertebral column stability and pain

Abbreviation used in this paper: $\mathrm{QOL}=$ quality of life. relief. Certain tumors such as renal cell warrant special consideration and preparation because of their vascularity.

\section{Patient Assessment}

General clinical, oncological, and spinal evaluations should be performed. It is essential to obtain a thorough history and perform a physical examination in patients with metastatic disease. The nature of the pain associated with osseous metastasis is often predictable. It is generally progressive and unrelenting, generally less related to activity than mechanical back pain, and often awakens the patient from sleep. Thorough systemic and medication reviews are essential for assessing perioperative risk and optimizing the patient's perioperative status. A review of the patient's history with regard to smoking status, previous malignancies, recent screening tests such as mammography or colonoscopy, suspicious skin lesions, weight loss, and change in bowel or bladder habits is essential. A physical examination focusing on skin, breasts, prostate, thyroid, lymph nodes, and stool guaiac may help narrow the search for a primary cause. Chronic steroid medication use and recent chemotherapy must be factored into the management.

Laboratory tests should include complete blood counts, erythrocyte sedimentation rate, basic chemistries, clotting parameters, postate-specific antigen, and serum and urine electrophoresis (to rule out myeloma). Urinalysis may reveal hematuria, which may be indicative of a primary renal or bladder cancer. An estimation of nutritional status 
may guide supplementation therapy to optimize wound healing and general recovery. Vigilant regard to the prevention, detection, and treatment of deep vein thrombosis is critical in debilitated patients.

\section{Preoperative Embolization}

Certain tumors warrant special consideration with regard to preoperative preparation to reduce intraoperative blood loss. Whereas preoperative treatment of renal cell metastases is the classic example, embolization has also been reported to be useful in other tumors including metastatic thyroid carcinoma. ${ }^{1,6}$ The intraoperative blood loss associated with renal cell can be substantial, sometimes necessitating that the procedure be abandoned. ${ }^{22}$ Preoperative embolization is not without risks to the arterial vessels and spinal cord, however, because it may be limited by involvement of major branches feeding the spinal cord. Additionally, induction of general anesthesia is often required. King, et al., ${ }^{9}$ reported their experience with renal cell metastasis and embolization. There was no intergroup difference in blood loss between those in whom embolization was performed (14 patients) and those in whom it was not (29 patients); however, surgery was aborted secondary to excessive blood loss in three of the nonembolized patients. Subsequently, embolization was performed in these three patients, and reoperation was thereafter successful.

\section{Pain Management}

Inadequate pain relief has a significant effect on a patient's functional capacity and QOL. In the treatment of these patients multimodality control of pain must be a priority; a combination of systemic therapy (analgesic medication, hormones, chemotherapeutic agents, steroids, bisphosphonates, and radiopharmaceuticals) and local therapy (surgery, nerve blocks, and external-beam radiotherapy) should be instituted. Inadequate pain control is a common concern among patients and physicians. The reasons for this shortcoming are multifactorial and include poor pain assessment, underutilization of adjuvant therapy, inadequate management of side effects, and reluctance to initiate maximum analgesia including opioids until the late stages of the disease. ${ }^{?}$

Analgesic therapy requires that assessment of pain relief be ongoing. Choices in medication must reflect a consideration of the severity and duration of a patient's symptoms. It is beneficial to involve subspecialists with extensive experience in the management of cancer-related pain early in the course of treatment. Nonsteroidal agents are often used in cases of mild pain and in combination with opioid drugs in cases involving more significant pain, but they have dose-limiting toxicity and implications regarding coagulation.

The use of sustained-release opiates allows for the maintenance of a more even pain control and can be supplemented with immediate-release preparations for breakthrough pain. Transcutaneous administration is best used when the dose providing stable control has been determined, because titration of the dose can be difficult. ${ }^{7}$ Other considerations include epidural and intrathecal administration. Cordotomy can also be considered in cases of otherwise intractable pain.

Steroid Therapy. Systemic steroids are commonly used in the management of pain in patients with spinal metastases. ${ }^{11,12,15}$ Steroids administered immediately after diagnosis may improve pain relief and possibly improve neurological function. Experimental evidence demonstrated in animal models indicates that systemic steroid therapy reduces spinal cord edema from epidural tumor and improves neurological outcome. ${ }^{26,27}$ The optimum dosing schedule is not well defined. The conventional therapeutic prescription is an initial 10-mg dose of dexamethasone followed by $4 \mathrm{mg}$ every 6 hours. There is some evidence that larger initial doses (100-mg bolus followed by 16-mg doses every 6 hours) provides better pain relief in preparation for definitive therapy. ${ }^{4}$ Some authors advocate using the spinal cord injury-related Solu-Medrol dosing (30- $\mathrm{mg} / \mathrm{kg}$ load and then $5.4 \mathrm{mg} / \mathrm{kg} /$ hour for 23 hours) in patients who present with rapid-onset neurological symptoms or for use intraoperatively.

Bisphosphonate Therapy. Bisphosphonates are structural analogs of endogenous pyrophosphate and their phosphate-carbon-phosphate nucleus binds strongly to mineralized bone matrix. Their principal effect is thought to be osteoclast inhibition and decreased bone resorption. They are used clinically, most commonly in cases of breast cancer and multiple myeloma, to reduce bone metastasis-related complications, especially that of pain. Two placebo-controlled randomized trials were recently conducted to evaluate the effects of bisphosphonates (pamidronate) on bone metastasis-related complications including pain, pathological fractures (vertebral and nonvertebral), hypercalcemia, spinal cord compression, and need for radiotherapy or surgery. ${ }^{5,24}$ Overall, the authors of both trials demonstrated a statistically significant reduction in mean skeleton-associated morbidity rate (number of events/year over a 2-year period). No significant reduction in vertebral fractures or spinal cord compression was shown in either trial, although a significant reduction in hypercalcemia was found in both. When pamidronate was used in conjunction with chemotherapy, Hortobagyi, et al. ${ }^{5}$ found a significant reduction in the overall incidence of fracture, as well as the need for surgery and/or radiosurgery (for pain or other indication). In the other study by the same group,${ }^{24}$ in which pamidronate was used in combination with hormone therapy, they found no statistical significance in these categories.

There is also recent evidence suggesting that bisphosphonates may have antiapoptotic and antiproliferative effects on macrophages and tumor cells in addition to osteoclasts. ${ }^{18}$ Three randomized trials were designed to address the potential antitumor effects. ${ }^{3,16,17}$ Unfortunately, the results among the trials have been inconsistent. Diel, et al., ${ }^{3}$ found statistically significant reductions in bone and visceral metastases as well as an increased rate of survival in clodronate-treated breast cancer patients with bone marrow involvement. Powles, et al. ${ }^{16}$ demonstrated an overall reduction in bone metastases but no changes in the rates of visceral metastasis or overall survival in a multicenter trial of over 1000 patients without risk selection criteria for metastasis. In a third trial in which investigators examined the effects of clodronate in 299 patients with nodal-positive breast cancer no benefit was shown in bone metastasis, visceral metastasis, or overall survival rates. ${ }^{17}$ Although the reason for these inconsistencies is unclear because the treatment doses were similar, it may 
be related to methodological differences such as inclusion criteria or numbers of patients. ${ }^{2}$ Finally, the effects of bisphosphonates on postoperative bone healing have not been evaluated.

Radiopharmaceutical Therapy. Radiopharmaceutical therapy with agents such as strontium-89 and samarium153 conjugated to pyrophosphate has demonstrated significant reduction in metastatic bone-related pain $^{3,19,20,25}$ and may ultimately improve a patient's mobility, reduce dependence on narcotic and nonnarcotic analgesics, improve performance status and QOL, and possibly improve survival rates. Radiopharmaceuticals are intended to localize to bone and reduce systemic exposure and toxicity. Radiation is concentrated predominantly at the metastatic site with lower exposure to normal bone marrow. ${ }^{7}$

\section{CONCLUSIONS}

Although surgery and radiotherapy remain the mainstay therapies in cases of symptomatic metastatic spinal disease, numerous adjuvant therapies are available that will improve QOL and perhaps ultimately extend survival. Adequate pain control is a major concern among patients, families, and physicians because it significantly affects functional capacity and QOL. Multimodality control of pain through a combination of systemic therapy (analgesics, hormones, chemotherapy, steroids, bisphosphonates, and radiopharmaceuticals) and local therapy (surgery, nerve blocks, and external-beam radiotherapy) must be a priority in the treatment of these patients. Further investigations are required to determine optimum combinations of these therapies in the treatment of metastatic spinal tumors. The patient's general medical condition including nutrition should be aggressively treated. Additionally, in our experience, preoperative embolization in cases of renal cell metastasis is beneficial.

\section{References}

1. Broaddus WC, Grady MS, Delashaw JB Jr, et al: Preoperative superselective arteriolar embolization: a new approach to enhance resectability of spinal tumors. Neurosurgery 27: 755-759, 1990

2. Diel IJ: Bisphosphonates in the prevention of bone metastases: current evidence. Semin Oncol 28 (4 Suppl 11):75-80, 2001

3. Diel IJ, Solomayer EF, Costa SD, et al: Reduction in new metastases in breast cancer with adjuvant clodronate treatment. $\mathbf{N}$ Engl J Med 339:357-363, 1998

4. Greenberg HS, Kim JH, Posner JB: Epidural spinal cord compression from metastatic tumor: results with a new treatment protocol. Ann Neurol 8:361-366, 1980

5. Hortobagyi GN, Theriault RL, Porter L, et al: Efficacy of pamidronate in reducing skeletal complications in patients with breast cancer and lytic bone metastases. Protocol 19 Aredia Breast Cancer Study Group. N Engl J Med 335:1785-1791, 1996

6. Jackson RJ, Gokaslan ZL, Loh SC: Metastatic renal cell carcinoma of the spine: surgical treatment and results. J Neurosurg 94 (Spine 1): 18-24, 2001

7. Janjan N: Bone metastases: approaches to management. Semin Oncol 28 (4 Suppl 11):28-34, 2001

8. Katagiri H, Takahashi M, Inagaki J, et al: Clinical results of nonsurgical treatment for spinal metastases. Int J Radiat Oncol Biol Phys 42:1127-1132, 1998

9. King GJ, Kostuik JP, McBroom RJ, et al: Surgical management of metastatic renal carcinoma of the spine. Spine 16:265-271, 1991

10. Kuban DA, el-Mahdi AM, Sigfred SV, et al: Characteristics of spinal cord compression in adenocarcinoma of prostate. Urology 28:364-369, 1986

11. Landreneau FE, Landreneau RJ, Keenan RJ, et al: Diagnosis and management of spinal metastases from breast cancer. J Neurooncol 23:121-134, 1995

12. Maranzano E, Latini $P$, Checcaglini F, et al: Radiation therapy in metastatic spinal cord compression. A prospective analysis of 105 consecutive patients. Cancer 67:1311-1317, 1991

13. Menda Y, Bushnell DL, Williams RD, et al: Efficacy and safety of repeated samarium-153 lexidronam treatment in a patient with prostate cancer and metastatic bone pain. Clin Nucl Med 25:698-700, 2000

14. Ortiz Gomez JA: The incidence of vertebral body metastases. Int Orthop 19:309-311, 1995

15. Osborn JL, Getzenberg RH, Trump DL: Spinal cord compression in prostate cancer. J Neurooncol 23:135-147, 1995

16. Powles TJ, McCloskey E, Paterson AH, et al: Oral clodronate and reduction in loss of bone mineral density in women with operable primary breast cancer. J Natl Cancer Inst 90: 704-708, 1998

17. Saarto T, Blomqvist C, Virkkunen P, et al: Adjuvant clodronate treatment does not reduce the frequency of skeletal metastases in node-positive breast cancer patients: 5-year results of a randomized controlled trial. J Clin Oncol 19:10-17, 2001

18. Senaratne SG, Pirianov G, Mansi JL, et al: Bisphosphonates induce apoptosis in human breast cancer cell lines. Br J Cancer 82:1459-1468, 2000

19. Serafini AN: Systemic metabolic radiotherapy with samarium153 EDTMP for the treatment of painful bone metastasis. Q J Nucl Med 45:91-99, 2001

20. Serafini AN: Therapy of metastatic bone pain. J Nucl Med 42:895-906, 2001

21. Smith EM, Hampel N, Ruff RL, et al: Spinal cord compression secondary to prostate carcinoma: treatment and prognosis. J Urol 149:330-333, 1993

22. Sundaresan N, Choi IS, Hughes JE, et al: Treatment of spinal metastases from kidney cancer by presurgical embolization and resection. J Neurosurg 73:548-554, 1990

23. Tatsui H, Onomura T, Morishita S, et al: Survival rates of patients with metastatic spinal cancer after scintigraphic detection of abnormal radioactive accumulation. Spine 21:2143-2148, 1996

24. Theriault RL, Lipton A, Hortobagyi GN, et al: Pamidronate reduces skeletal morbidity in women with advanced breast cancer and lytic bone lesions: a randomized, placebo-controlled trial. Protocol 18 Aredia Breast Cancer Study Group. J Clin Oncol 17:846-854, 1999

25. Turner SL, Gruenewald S, Spry N, et al: Less pain does equal better quality of life following strontium-89 therapy for metastatic prostate cancer. Br J Cancer 84:297-302, 2001

26. Ushio Y, Posner R, Kim JH, et al: Treatment of experimental spinal cord compression caused by extradural neoplasms. J Neurosurg 47:380-390, 1977

27. Ushio Y, Posner R, Posner JB, et al: Experimental spinal cord compression by epidural neoplasm. Neurology 27:422-429, 1977

28. Wong DA, Fornasier VL, MacNab I: Spinal metastases: the obvious, the occult, and the impostors. Spine 15:1-4, 1990

Manuscript received October 22, 2001.

Accepted in final form November 6, 2001.

Address reprint requests to: James M. Schuster, M.D., Ph.D., Department of Neurosurgery, University of Pennsylvania, 330 South 9th Street, 4th Floor, Philadelphia, Pennsylvania 19107. email: schustej@uphs.upenn.edu. 